\title{
Taurine ameliorates hyperglycemia and dyslipidemia by reducing insulin resistance and leptin level in Otsuka Long-Evans Tokushima fatty (OLETF) rats with long-term diabetes
}

\author{
Kyoung Soo Kim ${ }^{1,7}$, Da Hee $\mathrm{Oh}^{2}$, Jung Yeon $\mathrm{Kim}^{4}$, \\ Bong Gn Lee ${ }^{3}$, Jeong Soon You ${ }^{5}$, \\ Kyung Ja Chang ${ }^{5}$, Hyunju Chung ${ }^{3}$, \\ Myung Chul Yoo', Hyung-In Yang ${ }^{1}$, \\ Ja-Heon Kang ${ }^{6}$, Yoo Chul Hwang ${ }^{2}$, \\ Kue Jeong Ahn ${ }^{2}$, Ho-Yeon Chung ${ }^{2}$ and \\ In-Kyung Jeong ${ }^{2,7}$
}

\author{
${ }^{1}$ East-West Bone and Joint Research Institute \\ Kyung Hee University Hospital at Gangdong \\ ${ }^{2}$ Department of Endocrinology \\ Kyung Hee University Hospital at Gangdong \\ ${ }^{3}$ Core Research Laboratory \\ Clinical Research Institute \\ Kyung Hee University Hospital at Gangdong \\ Kyung Hee University College of Medicine \\ Seoul 134-727, Korea \\ ${ }^{4}$ Department of Pathology \\ Inje University Sanggye Paik Hospital \\ Seoul 139-707, Korea \\ ${ }^{5}$ Department of Food and Nutrition \\ Inha University \\ Incheon 402-751, Korea \\ ${ }^{6}$ Department of Ophthalmology \\ Kyung Hee University Hospital at Gangdong \\ Kyung Hee University College of Medicine \\ Seoul 134-727, Korea \\ ${ }^{7}$ Corresponding authors: Tel, 82-2-440-6791; \\ Fax, 82-2-440-6799; E-mail, labrea46@yahoo.co.kr (K.S.K.) \\ Tel, 82-2-440-6126; Fax, 82-2-440-6799; \\ E-mail, jik1016@dreamwiz.com (I.K.J.) \\ http://dx.doi.org/10.3858/emm.2012.44.11.075
}

Accepted 8 August 2012

Available Online 21 September 2012

Abbreviations: ACC, acetyl-CoA carboxylase; AMPK, adenosine monophosphate-activated protein kinase; GSIS, glucose-stimulated insulin secretion; HDL-C, high-density lipoprotein cholesterol; H\&E, hematoxylin and eosin; LDL-C, low-density lipoprotein cholesterol; LETO, Long-Evans Tokushima Otsuka; OLETF, Otsuka Long-Evans
Tokushima Fatty; T2DM, type 2 diabetes mellitus; TC, total cholesterol; TG, triglyceride

\begin{abstract}
This study aimed to determine whether taurine supplementation improves metabolic disturbances and diabetic complications in an animal model for type 2 diabetes. We investigated whether taurine has therapeutic effects on glucose metabolism, lipid metabolism, and diabetic complications in Otsuka LongEvans Tokushima fatty (OLETF) rats with long-term duration of diabetes. Fourteen 50-week-old OLETF rats with chronic diabetes were fed a diet supplemented with taurine $(2 \%)$ or a non-supplemented control diet for 12 weeks. Taurine reduced blood glucose levels over 12 weeks, and improved OGTT outcomes at 6 weeks after taurine supplementation, in OLETF rats. Taurine significantly reduced insulin resistance but did not improve $\beta$-cell function or islet mass. After 12 weeks, taurine significantly decreased serum levels of lipids such as triglyceride, cholesterol, high density lipoprotein cholesterol, and low density lipoprotein cholesterol. Taurine significantly reduced serum leptin, but not adiponectin levels. However, taurine had no therapeutic effect on damaged tissues. Taurine ameliorated hyperglycemia and dyslipidemia, at least in part, by improving insulin sensitivity and leptin modulation in OLETF rats with long-term diabetes. Additional study is needed to investigate whether taurine has the same beneficial effects in human diabetic patients.
\end{abstract}

Keywords: diabetes mellitus, type 2; dyslipidemias; hyperglycemia; rats, Long-Evans; taurine

\section{Introduction}

Type 2 diabetes (T2DM) is a metabolic disease characterized by chronic hyperglycemia and dyslipi- 
demia, which are associated with a wide range of complications, such as retinopathy, nephropathy, neuropathy, and cardiovascular abnormalities (Hoogwerf et al., 2006). Although the exact mechanisms that contribute to diabetic complications remain unclear, it has recently been suggested that disequilibrium between toxic metabolites (e.g., advanced glycation end-products, protein kinase $\mathrm{C}$ activation), oxidative stress, and protective endogenous factors (e.g., insulin, vascular endothelial growth factor, platelet-derived growth factors, and adiponectin) may play a role in diabetic complications (Jeong and King, 2011).

In T2DM, hyperglycemia results from insulin resistance of peripheral tissue and relative insulin deficiency (Shulman, 2000). Meanwhile, chronic insulin resistance increases levels of circulating non-esterified ('free') fatty acids (NEFAs), which are lipotoxic to beta cells. The impairing effects of NEFAs on glucose-stimulated insulin secretion (GSIS) and insulin sensitivity have been demonstrated in diabetic patients, especially in obese individuals with a predisposition for T2DM (Leung et al., 2004). Lipid-induced insulin resistance and beta cell dysfunction can be explained by several mechanisms, including increased synthesis of lipid metabolites (ceramides and diacylglycerides) (Itani et al., 2002; Holland et al., 2007), chronic inflammation (Hotamisligil, 2006), endoplasmic reticulum (ER) stress (Ozcan et al., 2004), and oxidative stress (Evans et al., 2002). In addition, increased free fatty acids, triglycerides (TG), and low density lipoprotein cholesterol (LDL-C), and decreased high density lipoprotein cholesterol (HDL-C), are atherogenic in patients with T2DM.

Taurine (2-aminoethylsulphonic acid) is a nonprotein amino acid present in almost all animal tissues, most abundantly as a free intracellular amino acid in human cells (Kim et al., 2007). Due to its unique chemical structure, taurine is involved in numerous biological and physiological functions that confer important health benefits. Thus, taurine is a cytoprotective agent in a variety of tissues. It also modulates a variety of cellular functions, including antioxidation, modulation of ion movement, osmoregulation, modulation of neurotransmitters, and conjugation of bile acids. In addition, taurine is involved in retinal development and function (Lourenco and Camilo, 2002). Notably, the beneficial roles of taurine in the context of diabetes have been widely studied in recent years (Ito et al., 2012). Accumulating data demonstrate the effectiveness of taurine supplementation against type 1 diabetes, type 2 diabetes mellitus, and metabolic syndrome. Above all, taurine supplementation reduces diabetic complications, including retinopathy, nephropathy, neuropathy, atherosclerosis, and cardiomyopathy. However, these effects of taurine on diabetes have usually been studied in animal models with streptozotocin-induced diabetes, which mimics type 1 diabetes. Furthermore, taurine has been administered at the early stage of hyperglycemia in animal models, and its reportedly beneficial effects have therefore been primarily preventive in nature. The health benefits of taurine have not been studied as much during the chronic stage of diabetes in diabetic animal models, and have not been studied at all in a model of spontaneous T2DM, such as the Otsuka Long-Evans Tokushima Fatty (OLETF) rat.

In present study, we determined whether taurine has beneficial effects on glucose metabolism, lipid metabolism, and diabetic complications in the OLETF diabetic rat with long-term disease duration. Such a finding would suggest that taurine supplementation might provide health benefits in human diabetic patients currently receiving treatment, and might even help prevent complications of T2DM.

\section{Results}

\section{Effect of taurine on glucose metabolism in OLETF rat with long-term diabetes}

To test whether taurine improves glucose metabolism and lowers insulin resistance in OLETF rats that had diabetes for more than 30 weeks, the diet of OLETF rats was supplemented with taurine $(2 \%)$ for 12 weeks starting at 50 weeks of age. During this period, blood was collected from the tail vein each week and plasma glucose levels were measured after overnight fasting. Plasma glucose was significantly reduced in the taurine-supplemented (taurine) group compared with the unsupplemented (control) group, even though there was no difference between the taurine and control groups at some time points (Figure 1A). In particular, at 60 weeks of age, the glucose level was $>300 \mathrm{mg} / \mathrm{dl}$ in the control group, and approximately $200 \mathrm{mg} / \mathrm{dl}$ in the taurine group. In an attempt to explain the difference in glucose levels, we also measured food intake and body weight. However, food intake did not differ significantly between the two groups (Figure 1B). Body weights were also not significantly different (data not shown). These findings indirectly suggest that the reduced blood glucose in the taurine group resulted from the physiological effects of taurine itself, rather than from weight loss or reduced food intake.

To confirm that taurine enhanced glucose metabolism in diabetic rats, OGTTs were conducted in diabetic rats (Figure 2A). At the beginning of taurine supplementation, the level of glucose (indicated by 
A

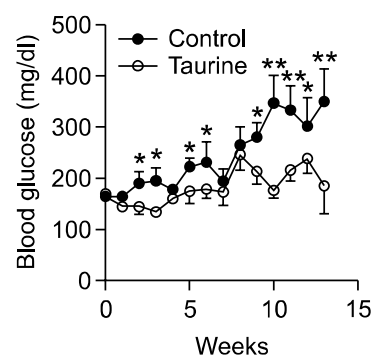

B

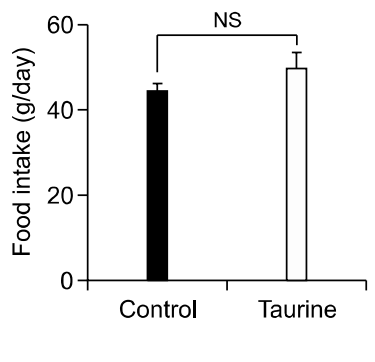

Figure 1. The effect of taurine supplementation on glucose metabolism in OLETF rats with long-term diabetes. A taurine (2\%)-supplemented diet was fed to OLETF rats for 12 weeks, beginning at 50 weeks of age. (A) Blood glucose levels during the 12-weeks supplementation period. (B) Comparison of food intake between taurine and control groups. Data are expressed as mean \pm standard error of the mean (SEM). Between-group differences were compared using the Mann-Whitney $U$ test. Differences were considered statistically significant at $P<0.05$. ${ }^{*} P<0.05$; ${ }^{* *} P<$ 0.01 ; NS, not significant.

the OGTT) was not different between the two groups; however, at 6 weeks, the serum glucose level was significantly lower in the taurine group than the control group at 90 and 120 min after glucose loading. At 12 weeks, the serum glucose level at baseline $(0 \mathrm{~min})$ was significantly lower in the taurine group than the control group, although serum glucose levels after oral glucose loading did not differ significantly between the two groups. This finding suggests that OLETF rats at 60 weeks of age have severe glucotoxicity due to beta cell destruction, which is not overcome by a taurine-supplemented diet.

As expected, the $\mathrm{HbA} 1 \mathrm{c}$ level of OLETF rats was significantly higher than that of age-matched nondiabetic LETO rats (data not shown). However, taurine did not significantly reduce the level of $\mathrm{HbA} 1 \mathrm{c}$ in diabetic OLETF rats after taurine feeding for 12 weeks (Figure 2B). These findings also indirectly suggest that the extent of the taurine-induced decrease in serum glucose level could not have led to the distinctive decrease in $\mathrm{HbA} 1 \mathrm{c}$.

\section{Effects of taurine on beta cell function and insulin resistance}

We wondered whether taurine supplementation would increase basal HOMA- $\beta$ and decrease HOMA-IR. Overnight fasting serum glucose and insulin levels were significantly reduced in the taurine group (Figure 3A). Taurine supplementation significantly reduced HOMA-IR as a marker of insulin resistance at 12 weeks, and also did not significantly increase HOMA- $\beta$ as a marker of basal beta cell function (Figure 3B). Insulin tolerance test showed that kitt $(\% / m i n)$ value of taurine group was significantly
A
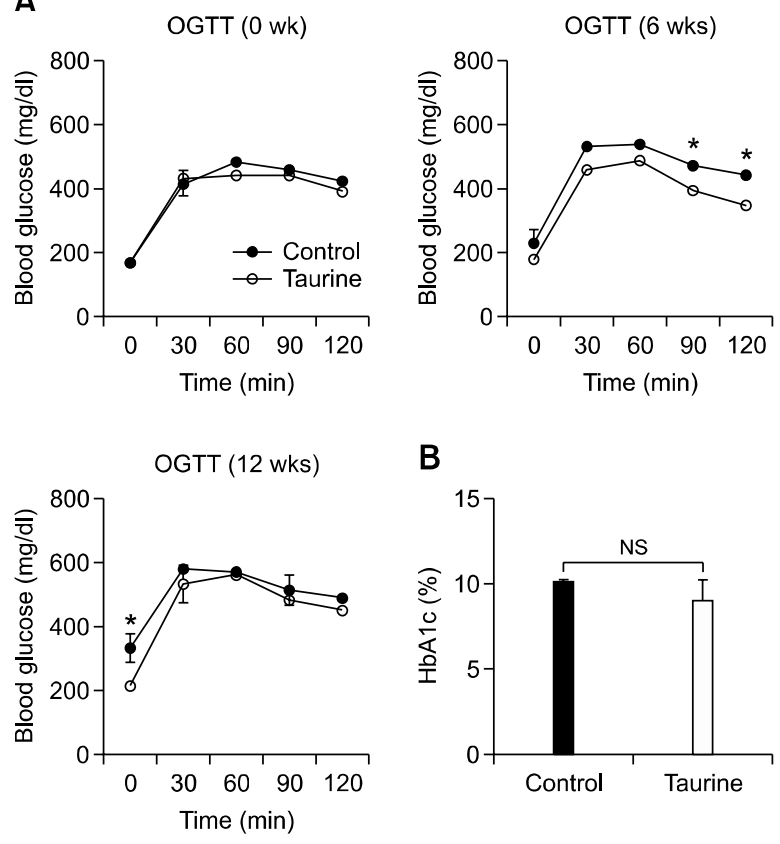

B

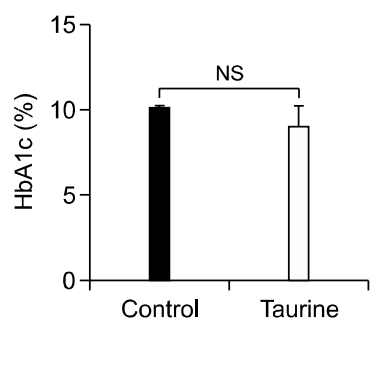

Figure 2. The effects of taurine supplementation on oral glucose tolerance tests (OGTTS) and glycated haemoglobin ( $\mathrm{HbA1C}$ ) in OLETF rats. (A) OGTTs were conducted at 0,6 , and 12 weeks after beginning the taurine-supplemented diet. (B) HbA1c levels. Data are expressed as the mean \pm standard error of the mean (SEM). Between-group differences were compared using the Mann-Whitney $U$ test. Differences were considered statistically significant at $P<0.05$. ${ }^{*} P<0.05$; NS, not significant.

higher than that of control group (Figure 3C).

\section{Effects of taurine on tissue targets of insulin secretion and action}

We wondered whether the taurine enhanced pancreatic beta cell mass and improved fatty change of liver or muscle. Pancreatic tissue was investigated by staining with $\mathrm{H} \& \mathrm{E}$ and by immunostaining with an anti-insulin antibody. Compared with normal LETO rats, islet cells in OLETF rats were mostly destroyed, and therefore few beta cells were detected by immunostaining with the anti-insulin antibody. The beta cell area of the taurine group did not differ significantly from that of the control group (Figure 4A).

Insulin resistance in liver, muscle, and adipose tissues has also been characterized in T2DM. The gross morphological changes in fat, liver, and muscle tissues were assayed histopathologically (Figure 4B). No specific differences between tissues in the taurine and the control group were noted in these tissues. Liver tissue showed normal architecture with a mildly dilated portal area, and with periportal and perisinusoidal fibrosis compared with the LETO control group. Fatty change and nuclear vacuolisation were not observed. 
A

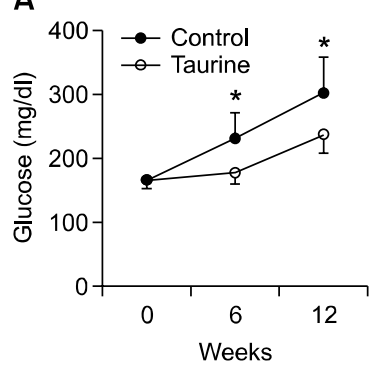

B

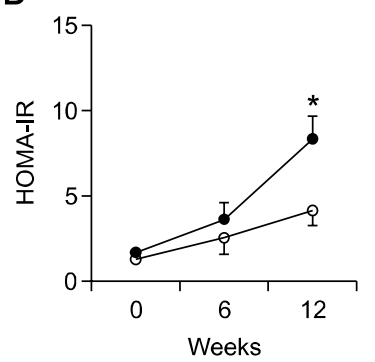

C

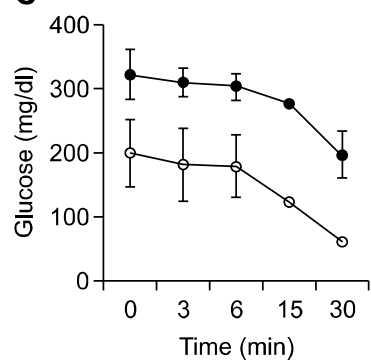

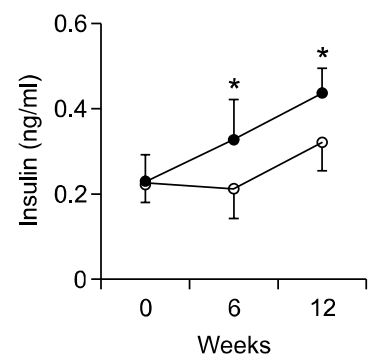
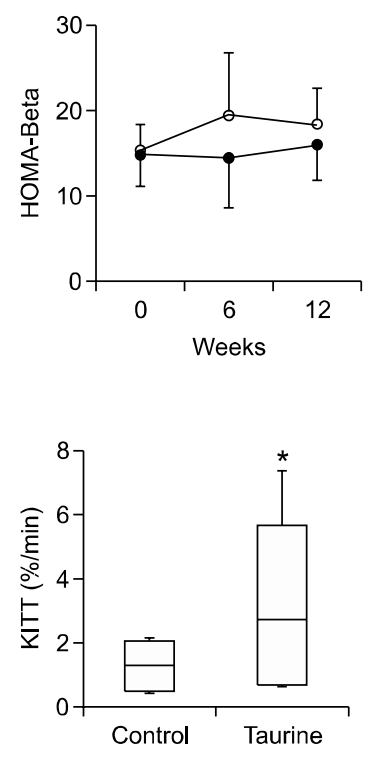

Figure 3. The effects of taurine supplementation on insulin resistance (HOMA-IR) and beta cell function (HOMA- $\beta$ ) in OLETF rats. (A) Levels of glucose and insulin 0, 6, and 12 weeks after beginning the taurine-supplemented diet. (B) Comparison of HOMA-IR and HOMA- $\beta$ between taurine and the control groups. (C) Comparison of Insulin Tolerance Test (ITT) between two groups. Between-group differences were compared using the Mann-Whitney $\mathrm{U}$ test. Differences were considered statistically significant at $P<0.05 .{ }^{*} P<0.05$; NS, not significant.

\section{Effects of taurine on serum lipid profile and adipokine levels}

To examine whether a taurine-supplemented diet would improve lipid metabolism in diabetic rats, fasting serum was analysed for levels of TG, TC, HDL-C, and LDL-C at the time of sacrifice (after rats were fed a taurine diet for 12 weeks). Serum levels of TG, TC, HDL-C, and LDL-C were significantly decreased in the taurine group compared with the control group (Figure 5A). In addition, adipokines such as adiponectin and leptin are important factors that affect glucose and lipid metabolism. Therefore, we also studied whether a taurine supplement would affect serum adiponectin or serum leptin in diabetic OLETF rats (Figure $5 \mathrm{~B}$ ). Taurine supplementation
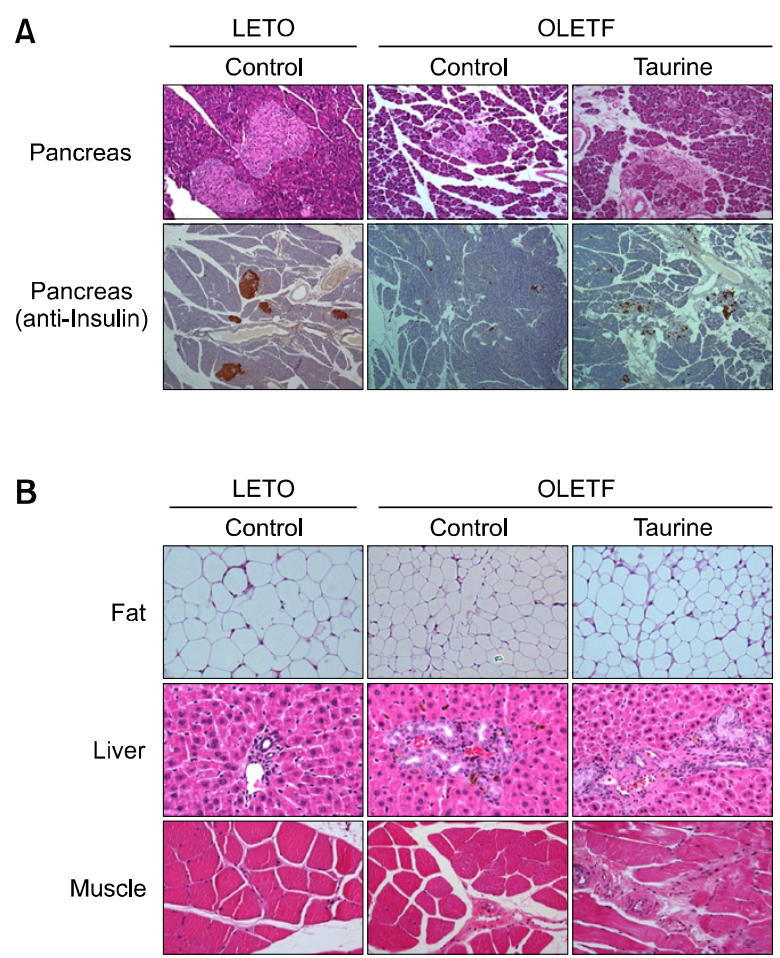

Figure 4. The effects of taurine supplementation on tissue targets of insulin secretion and action in OLETF rats. (A) Pancreatic tissue was stained by H\&E (upper panel) and anti-insulin antibody (lower panel) (Magnification: H\&E stain, 40×; Immunostaining, 10×) . (B) Fat, liver, and muscle tissues were stained by H\&E after adhering to a taurine diet for 12 weeks (H\&E stain, $100 \times$ magnification).

did not affect serum adiponectin; however, serum leptin was significantly reduced in OLETF rats in the taurine group compared with the control group.

\section{Effect of taurine on diabetic vascular complication}

Because taurine supplementation had beneficial effects on glucose and lipid metabolism in this study, we examined whether taurine supplementation had therapeutic effects on tissues that had been negatively affected by diabetes. Heart, aorta, and kidney tissues were investigated histopathologically. Compared with normal LETO rats, diabetic OLETF rats exhibited prominent nodular glomerulosclerosis in kidney tissue; the tissue damage was not reversed by taurine supplementation. Consistent with the kidney damage observed in OLETF rats, urinary albumin levels did not differ significantly between taurine and control groups (data not shown). Atherosclerosis is another hallmark of diabetes. We did not observe lesions of myocardial infarction, coronary atherosclerosis, or arteriosclerosis in the aorta and heart in OLETF rats. 
A
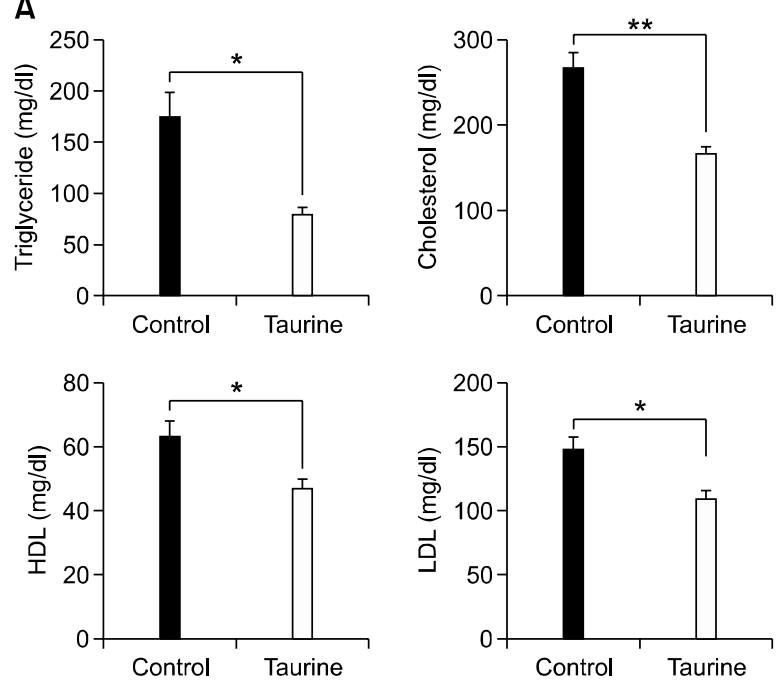

B
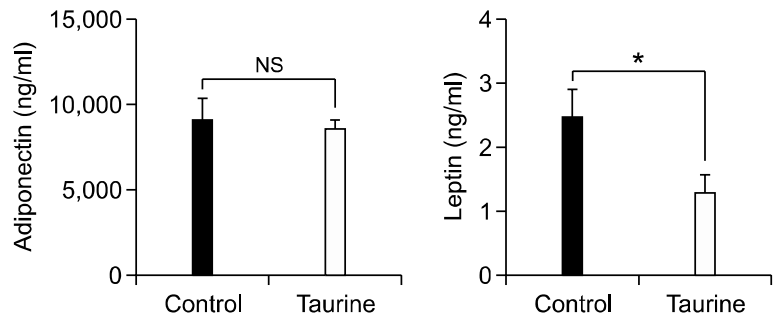

Figure 5. The effects of taurine supplementation on serum lipid and serum adipokine levels in OLETF rats. (A) Levels of triglycerides (TG), cholesterol, high density lipoprotein cholesterol (HDL-C) and low density lipoprotein cholesterol (LDL-C). (B) Serum adiponectin and leptin levels in OLETF rats after adhering to a taurine diet for 12 weeks. Between-group differences were compared using the Mann-Whitney $U$ test. Differences were considered statistically significant at $P<0.05$. ${ }^{*} P<0.05$; ${ }^{* *} P<$ 0.01 ; NS, not significant.

\section{Discussion}

In this study, 12 weeks of taurine supplementation significantly improved insulin sensitivity and lowered serum glucose and serum lipid concentrations in diabetic OLETF rats that were 50 weeks old at the beginning of supplementation. However, the taurineinduced reductions in serum glucose and lipid levels did not alleviate the diabetic complications that had already been induced by hyperglycemia, which had persisted for at least 30 weeks. These results suggest that even though taurine supplementation was able to alleviate the hyperglycemia and dyslipidemia, it was too late to reverse the diabetic nephropathy. Furthermore, 12 weeks of taurine supplementation was not enough time to delay the progression of complications. Most studies regarding taurine's effects on diabetes have been conducted in streptozotocin-induced type 1 diabetes animal

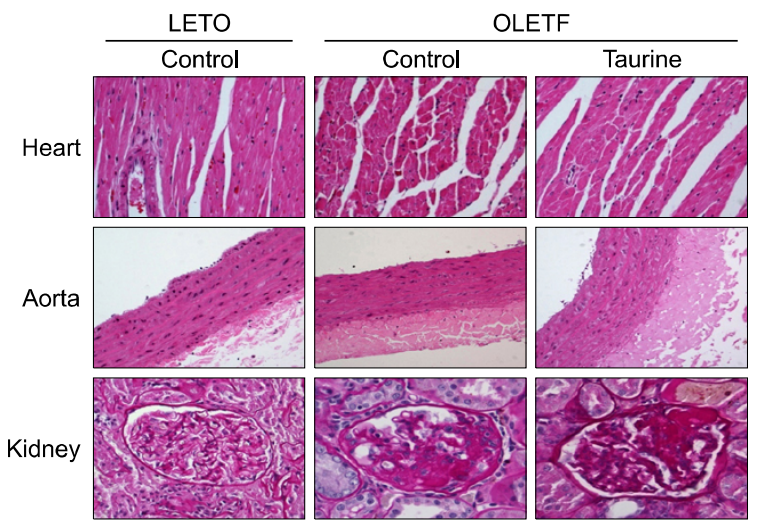

Figure 6. The effects of taurine supplementation on various tissues that have been negatively affected by diabetes. Heart, aorta, and kidney tissues were $\mathrm{H} \& \mathrm{E}$ stained, and the degree of tissue damage was compared between LETO and OLETF rats after adhering to a taurine diet for 12 weeks (H\&E stain; Magnification: $200 \times$ for kidney, $100 \times$ for heart and aorta).

models, or in early stage of animal models for T2DM. The results of the present study suggest that taurine supplementation may provide important health benefits in diabetes patients.

To further expand the use of taurine as a therapeutic tool with which to treat diabetic patients, the molecular mechanisms by which taurine lowers serum glucose and serum lipid levels should be elucidated. Many other studies have demonstrated that taurine has therapeutic effects on diabetes in various animal models. Taurine supplementation has been shown to suppress hyperglycemia and reduce plasma $\mathrm{HbA} 1 \mathrm{c}$, cholesterol, and triglycerides in streptozotocin-induced type 1 diabetic rats (Tokunaga et al., 1983). It has also been shown to suppress hyperglycemia and insulin resistance in a high fructose-fed rat model (El Mesallamy et al., 2010), and has improved insulin secretion and insulin sensitivity in acute glucose or lipid infusion models (Haber et al., 2003). However, daily supplementation with $1.5 \mathrm{~g}$ taurine for 8 weeks had no effect on insulin secretion, insulin sensitivity, or blood lipid levels in overweight men with a genetic predisposition for T2DM, suggesting that dietary taurine supplementation may not prevent the development of T2DM in humans (Brons et al., 2004). Taurine has also exhibited a beneficial effect on the islet dysfunction induced by free fatty acids in Wistar rats (Oprescu et al., 2007). These beneficial effects of taurine appear to be mostly based on the antioxidant activity of taurine, as well as on various protective effects against high glucose exposure in cultured beta cells (e.g., the modulation of mitochondrial calcium handling and the stabilization of protein folding) (Han et al., 2004; Kaniuk et al., 2007). 
To further elucidate the physiological mechanisms by which taurine exerts its therapeutic effects on glucose and lipid levels, we examined serum adiponectin and leptin levels in diabetic rats because adipokines are involved in energy homeostasis and the regulation of glucose and lipid metabolism, immunity, neuroendocrine function, and cardiovascular function. Their physiological functions were welldescribed in a previous report (Gulcelik et al., 2009). In particular, adiponectin has been suggested to have insulin-sensitizing, anti-inflammatory, and antiatherogenic effects. The actions of adiponectin in suppressing gluconeogenesis and enhancing lipid oxidation are related to the activation of adenosine monophosphate-activated protein kinase (AMPK) and the inhibition of acetyl-CoA carboxylase (ACC) in liver and muscle (Yamauchi et al., 2002). Adiponectin increases fatty acid combustion and energy consumption (and decreases tissue triglyceride content) in muscle and liver. Through these mechanisms, adiponectin contributes to improved insulin-induced signal transduction, and hence improved insulin sensitivity (Yamauchi et al., 2001).

Leptin is an adipose-derived hormone that is involved in appetite and energy metabolism. Leptin has also been shown to be associated with insulin resistance and inflammatory factors. In T2DM, insulin resistance is reportedly associated with elevated leptin levels independent of body fat mass (Segal et al., 1996). In the present study, adiponectin levels were unchanged; however, leptin levels were significantly reduced in the taurine group. Therefore, the reduction in serum lipid and glucose levels was not linked to any change in adiponectin level. We hypothesize that the reduction in leptin may be involved in improving insulin sensitivity in the taurine group. In many cases, leptin was significantly decreased after weight loss (Masquio et al., 2012). However, body weight and epididymal fat mass did not differ significantly between the taurine and control groups in the present study. This study is the first to evaluate the effect of taurine on leptin level, and additional studies will be needed to elucidate the possible effect of taurine on leptin signaling in adipose tissue.

To explain the mechanisms by which the serum lipid level was reduced in the taurine group, we investigated the gene expression of AMPK, ACC, stearoyl-CoA desaturase 1 , hydroxymethyl glutaryl CoA reductase, and fatty acid synthase, all of which are involved in lipogenesis in liver tissue, using RT-PCR and Western blotting (data not shown) (Kim et al., 2009). We did not observe consistent and significant taurine-induced changes in gene expression. Nakaya et al. showed similar data (Nakaya et al., 2000). They showed the decreased level of cholesterol and triglyceride after taurine treatment, but they did not show the level of LDL-C, or HDL-C. They suggested that taurine increased secretion of cholesterol into bile acid. Murakami et al. also showed taurine decreased cholesterol by enhancing the activity of cholesterol 7-alpha monooxygenase, a rate-limiting enzyme in bile acid synthesis and stimulated bile acid production (Murakami et al., 1996). Therefore, taurine may decrease cholesterol catabolism rather than cholesterol synthesis. Taurine administration prevented fatty livers as a result of Kupffer celldependent injury (Bruns et al., 2011) and reduced hepatic steatosis in rats (Chen et al., 2009).

Previous studies have suggested that taurine supplementation has therapeutic and protective effects on tissues that have been damaged by diabetes (Ito et al., 2012). Recently, it was demonstrated that taurine supplementation can play a beneficial role in regulating diabetes and its associated heart complications in alloxan-induced diabetes (Das et al., 2012). Furthermore, in OLETF rats, taurine acted as an antioxidant to attenuate islet fibrosis, which is important in the progression of pancreatic beta cell failure and is induced by oxidative stress in T2DM (Lee et al., 2011). However, in the present study, taurine supplementation did not reverse tissue damage (pancreas, heart, aorta, and kidney) associated with long-term diabetes in OLETF rats. Meanwhile, taurine improved vascular endothelial dysfunction induced by experimental type 1 diabetes; this effect might be associated with the downregulation of lectin-like oxidised LDL receptor-1 and intercellular adhesion molecule 1 expression on aortic vascular endothelium via taurine's antioxidative property (Wang et al., 2008). Taurine supplementation also protected against diabetic rat endothelial dysfunction by preventing upregulation of endothelial nitric oxide synthase mRNA (Ikubo et al., 2011). Other potential pathways may also be involved in the protective action of taurine against hyperglycemia-induced endothelial dysfunction. Taurine may inhibit the production of advanced glycated end-products and oxidized LDL by malondialdehyde and hypochlorous acid scavenging, hypochlorous acid-dependent nitric oxide reduction, and leukocyte-endothelium interactions (Ito et al., 2012).

The hypothesized mechanisms underlying taurine's effects on diabetes are based on the antioxidant action of taurine. Diabetes is thought to be induced by reactive oxygen species, generated in the mitochondria of glucose-treated cells, which promote reactions leading to the development of diabetic complications. The generation of oxidants in diabetes is attributed to impaired glucose and fatty acid 
metabolism. Diabetes is also associated with a decline in the levels of the endogenous antioxidant taurine in a number of tissues, raising the possibility that changes in taurine status might also contribute to the severity of oxidant-mediated damage. The antioxidant actions of taurine have been well described elsewhere (Schaffer et al., 2009). Taurine appears to play important roles in detoxifying hypochlorous acid in vivo, and is necessary for forming taurine-conjugated tRNAs in the mitochondria, which are also required for normal translation of mitochondrial-encoded proteins. Thus, taurine deficiency decreases the flux through the electron transport chain, which in turn creates a dysfunctional oxygen respiratory pathway, which leads to excess superoxide anions. Restoration of taurine levels increases the levels of conjugated tRNA, restores respiratory chain activity, and increases adenosine triphosphate synthesis at the expense of superoxide anion production. In addition, taurine depletion caused by hyperglycemia may provide a link between mitochondrial dysfunction and diabetes (Hansen et al., 2010).

In this study, taurine ameliorated hyperglycemia and dyslipidemia by improving insulin resistance and decreasing leptin secretion in OLETF rats with long-term diabetes. Although diabetic nephropathy and atherosclerosis were not reversed by taurine supplementation in long-term diabetic animals, taurine may confer its metabolically beneficial effects onto diabetic patients. Additional studies are required to determine whether taurine treatment has the same positive effects on diabetes in humans as have been shown in this animal model.

\section{Methods}

\section{Animals and experimental design}

Male OLETF rats were maintained in plastic cages at $21^{\circ} \mathrm{C}$ to $24^{\circ} \mathrm{C}$ on a $12 \mathrm{~h}$ light/dark cycle, and were given free access to pellet food and water until the age of 50 weeks. They were randomly assigned to two groups of seven rats each: one group received a taurine-supplemented diet (taurine-supplemented group) and the other did not receive supplementation (unsupplemented group). The experimental diet was based on AIN76 (Bieri et al., 1977). Taurine (2\%) was added to the food of the taurine-supplemented group between 50 and 62 weeks of age. Supplemental Data Table S1 shows the composition of the taurine diet. Body weight and fasting blood glucose concentrations did not differ significantly between the groups at the beginning of the experiments. Nondiabetic LETO (Long-Evans Tokushima Otsuka) rats fed the same standard diet were used as age-matched controls (nondiabetic group). Blood was collected from tail veins every week during the experimental period. On the last day of the experimental period, blood was collected from the abdominal aorta to assay insulin, lipid, and glucose levels. All methods were approved by the Animal Care and Use Committee of Kyung Hee University Hospital at Gangdong. All procedures were conducted in accordance with the Guide for the Care and Use of Laboratory Animals, published by the Korean National Institute of Health.

\section{Oral glucose tolerance test}

Rats underwent an oral glucose tolerance test (OGTT) after an overnight fast. Rats were given a glucose solution $(2.0 \mathrm{~g} / \mathrm{kg}$, p.o.). For glucose and insulin measurements, blood samples were collected from the tail vein at 0 min (just before the oral administration of glucose), and at 30 , 60 , and 120 min after glucose loading.

\section{Insulin tolerance test}

Insulin tolerance test was performed after overnight fasting. After fasting glucose was measured by glucometer, human regular insulin $(0.1 \mathrm{U} / \mathrm{kg}$, Lilly $)$ was injected intraperitoneally. And blood glucose level was measured at $0,3,6,9,12,15,30 \mathrm{~min}$ after i.p. injection of regular insulin. Rate constant for ITT (KITT) was calculated using formula KITT $(\% / \mathrm{min})=0.693 / \mathrm{t}^{1 / 2} \times 100$, where $\mathrm{t}^{1 / 2}$ was calculated from the slope of the glucose concentration during the period from 3 to $15 \mathrm{~min}$ after insulin injection.

\section{Laboratory analysis of blood samples}

Fasting blood samples were collected for the measurement of fasting plasma glucose, fasting plasma insulin, glycated haemoglobin (HbA1c), lipid profiles, and adipokines. Plasma glucose levels were determined by blood glucose sensors (MediSense Optium Xceed) and electrodes (Optium, Abbott Diabetes Care Inc., Alameda, CA). HbA1c levels were measured using a Siemens DCA Vantage ${ }^{\mathrm{TM}}$ Analyzer (Tarrytown, NY). For serum analysis of insulin, adiponectin, and leptin levels, serum was obtained by centrifugation of blood $\left(10,000 \times g, 10 \mathrm{~min}, 4^{\circ} \mathrm{C}\right)$ and assayed using an ELISA Kit (Millipore, Billerica, MA). Serum TG, total cholesterol (TC), and HDL-C levels were determined using an automatic analyser (BPC BioSed srl, Italy). After the precipitation of low-density lipoprotein (LDL) and very low-density lipoprotein for $10 \mathrm{~min}$ at $3000 \mathrm{rpm}$ (HM $150 \mathrm{IV}$, Hanil Industrial Co., Korea) (Warnick and Albers, 1978), HDL-C was obtained from whole serum with a high density lipoprotein precipitation reagent (AM204-1, Asan Pharmaceutical, Korea). The precipitate was analysed for HDL-C using the same method as for TC. Serum LDL-C values were calculated using the Friedewald formula (Friedewald et al., 1972) as follows: LDL-C = TC - (HDL-C + TG/5). Standard serum (lot no. 053801, Asan Pharmaceutical, Korea) was used for calibration before each parameter was analysed. Results are expressed as $\mathrm{mg} / \mathrm{dl}$ serum.

Homoeostasis model assessment was used to estimate basal beta cell function (HOMA- $\beta$ ) and insulin resistance (HOMA-IR). The following equations were used to calculate beta cell function and insulin resistance: $\mathrm{HOMA}-\beta=20$ $\times$ fasting plasma insulin $(\mu \mathrm{U} / \mathrm{ml}) /[$ fasting plasma glucose $(\mathrm{mmol} / \mathrm{L})-3.5]$, HOMA-IR $=[$ Fasting plasma insulin $(\mu \mathrm{U} / \mathrm{ml})$ $\times$ Fasting plasma glucose $(\mathrm{mmol} / \mathrm{L})] / 22.5$. 


\section{Histopathology}

Fat, heart, liver, pancreas, muscle, aorta, and kidney tissue were obtained from each rat. The tissues of each rat group were histologically examined. Tissues were fixed with $10 \%$ buffered formalin, and paraffin-embedded sections were stained with hematoxylin and eosin (H\&E). Insulin, periodic acid-Schiff, pethenamine silver, and Masson's trichrome staining were also completed to clearly visualise pancreatic beta cells, blood vessels, and collagen, respectively.

\section{Statistical analysis}

Experimental data are expressed as mean \pm standard error of the mean (SEM). Between-group differences were compared using the Mann-Whitney test. Prism software v. 4 (Graphpad Software, San Diego, CA) was used for statistical analysis and graphing. Differences were considered statistically significant at $P<0.05$.

\section{Supplemental data}

Supplemental data include a table and can be found with this article online at http://e-emm.or.kr/article/article_files/ SP-44-11-04.pdf.

\section{Acknowledgements}

This research was supported by the Industrialization Support Program for Bio-technology of Agriculture and Forestry (810002031SB110 and 810002032WT311), Ministry for Food, Agriculture, Forestry and Fisheries, Republic of Korea. This research was also supported by the Basic Science Research Program through the National Research Foundation of Korea (NRF), which is funded by the Ministry of Education, Science and Technology (grant numbers 2010-0024089 and 2011-0009061).

\section{Competing interests}

The authors declare that they have no competing interests.

\section{References}

Bieri J, Stoewsand G, Briggs G, Phillips, Woodard RJ, Knapka J. Report of the American Institute of Nurtition ad hoc Committee on Standards for Nutritional Studies. J Nutr 1977; 107:1340-8

Brons C, Spohr C, Storgaard H, Dyerberg J, Vaag A. Effect of taurine treatment on insulin secretion and action, and on serum lipid levels in overweight men with a genetic predisposition for type II diabetes mellitus. Eur J Clin Nutr 2004;58:1239-47

Bruns H, Watanpour I, Gebhard MM, Flechtenmacher C, Galli U, Schulze-Bergkamen H, Zorn M, Buchler MW, Schemmer P. Glycine and taurine equally prevent fatty livers from Kupffer cell-dependent injury: an in vivo microscopy study. Microcirculation 2011;18:205-13

Chen X, Sebastian BM, Tang H, McMullen MM, Axhemi A,
Jacobsen DW, Nagy LE. Taurine supplementation prevents ethanol-induced decrease in serum adiponectin and reduces hepatic steatosis in rats. Hepatology 2009;49: 1554-62

Das J, Vasan V, Sil PC. Taurine exerts hypoglycemic effect in alloxan-induced diabetic rats, improves insulin-mediated glucose transport signaling pathway in heart and ameliorates cardiac oxidative stress and apoptosis. Toxicol Appl Pharmacol 2012;258:296-308

El Mesallamy HO, El-Demerdash E, Hammad LN, El Magdoub HM. Effect of taurine supplementation on hyperhomocysteinemia and markers of oxidative stress in high fructose diet induced insulin resistance. Diabetol Metab Syndr 2010;2:46

Evans JL, Goldfine ID, Maddux BA, Grodsky GM. Oxidative stress and stress-activated signaling pathways: a unifying hypothesis of type 2 diabetes. Endocr Rev 2002;23:599-622

Friedewald WT, Levy RI, Fredrickson DS. Estimation of the concentration of low-density lipoprotein cholesterol in plasma, without use of the preparative ultracentrifuge. Clin Chem 1972;18:499-502

Gulcelik NE, Usman A, Gurlek A. Role of adipocytokines in predicting the development of diabetes and its late complications. Endocrine 2009;36:397-403

Haber CA, Lam TK, Yu Z, Gupta N, Goh T, Bogdanovic E, Giacca A, Fantus IG. N-acetylcysteine and taurine prevent hyperglycemia-induced insulin resistance in vivo: possible role of oxidative stress. Am J Physiol Endocrinol Metab 2003 285:E744-53

Han J, Bae JH, Kim SY, Lee HY, Jang BC, Lee IK, Cho CH, Lim JG, Suh SI, Kwon TK, Park JW, Ryu SY, Ho WK, Earm YE, Song DK. Taurine increases glucose sensitivity of UCP2overexpressing beta-cells by ameliorating mitochondrial metabolism. Am J Physiol Endocrinol Metab 2004;287: E1008-18

Hansen SH, Andersen ML, Cornett C, Gradinaru R, Grunnet $\mathrm{N}$. A role for taurine in mitochondrial function. J Biomed Sci 2010;17 Suppl 1:S23

Holland WL, Brozinick JT, Wang LP, Hawkins ED, Sargent KM, Liu Y, Narra K, Hoehn KL, Knotts TA, Siesky A, Nelson $\mathrm{DH}$, Karathanasis SK, Fontenot GK, Birnbaum MJ, Summers SA. Inhibition of ceramide synthesis ameliorates glucocorticoid-, saturated-fat-, and obesity-induced insulin resistance. Cell Metab 2007;5:167-79

Hoogwerf BJ, Sferra J, Donley BG. Diabetes mellitus-overview. Foot Ankle Clin 2006;11:703-15

Hotamisligil GS. Inflammation and metabolic disorders. Nature 2006;444:860-7

Ikubo N, Saito M, Tsounapi P, Dimitriadis F, Ohmasa F, Inoue S, Shimizu S, Kinoshita Y, Satoh K. Protective effect of taurine on diabetic rat endothelial dysfunction. Biomed Res 2011;32: 187-93

Itani SI, Ruderman NB, Schmieder F, Boden G. Lipid-induced insulin resistance in human muscle is associated with changes in diacylglycerol, protein kinase $\mathrm{C}$, and lkappaBalpha. Diabetes 2002;51:2005-11 
Ito T, Schaffer SW, Azuma J. The potential usefulness of taurine on diabetes mellitus and its complications. Amino Acids 2012;42:1529-39

Jeong IK, King GL. New perspectives on diabetic vascular complications: the loss of endogenous protective factors induced by hyperglycemia. Diabetes Metab J 2011;35:8-11

Kaniuk NA, Kiraly M, Bates H, Vranic M, Volchuk A, Brumell $\mathrm{JH}$. Ubiquitinated-protein aggregates form in pancreatic beta-cells during diabetes-induced oxidative stress and are regulated by autophagy. Diabetes 2007;56:930-9

Kim SJ, Gupta RC, Lee HW. Taurine-diabetes interaction: from involvement to protection. Curr Diabetes Rev 2007;3: 165-75

Kim DY, Yuan HD, Chung IK, Chung SH. Compound K, intestinal metabolite of ginsenoside, attenuates hepatic lipid accumulation via AMPK activation in human hepatoma cells. J Agric Food Chem 2009;57:1532-7

Lee E, Ryu GR, Ko SH, Ahn YB, Yoon KH, Ha H, Song KH. Antioxidant treatment may protect pancreatic beta cells through the attenuation of islet fibrosis in an animal model of type 2 diabetes. Biochem Biophys Res Commun 2011;414: 397-402

Leung N, Sakaue T, Carpentier A, Uffelman K, Giacca A, Lewis GF. Prolonged increase of plasma non-esterified fatty acids fully abolishes the stimulatory effect of $24 \mathrm{~h}$ of moderate hyperglycaemia on insulin sensitivity and pancreatic beta-cell function in obese men. Diabetologia 2004;47: 204-13

Lourenco R, Camilo ME. Taurine: a conditionally essential amino acid in humans? An overview in health and disease. Nutr Hosp 2002;17:262-70

Masquio DC, de Piano A, Sanches PL, Corgosinho FC, Campos RM, Carnier J, Silva PL, Caranti DA, Tock L, Oyama LM, Nascimento CM, de Mello MT, Tufik S, Damaso AR. The effect of weight loss magnitude on pro/anti-inflammatory adipokines and carotid intima-media thickness in obese adolescents engaged in interdisciplinary weight-loss therapy. Clin Endocrinol (Oxf) 2012 [Epub ahead of print]

Murakami S, Yamagishi I, Asami Y, Ohta Y, Toda Y, Nara Y, Yamori Y. Hypolipidemic effect of taurine in stroke-prone spontaneously hypertensive rats. Pharmacology 1996;52: 303-13

Nakaya Y, Minami A, Harada N, Sakamoto S, Niwa Y, Ohnaka M. Taurine improves insulin sensitivity in the Otsuka Long-Evans Tokushima Fatty rat, a model of spontaneous type 2 diabetes. Am J Clin Nutr 2000;71:54-8

Oprescu Al, Bikopoulos G, Naassan A, Allister EM, Tang C, Park E, Uchino H, Lewis GF, Fantus IG, Rozakis-Adcock M, Wheeler MB, Giacca A. Free fatty acid-induced reduction in glucose-stimulated insulin secretion: evidence for a role of oxidative stress in vitro and in vivo. Diabetes 2007;56: 2927-37

Ozcan U, Cao Q, Yilmaz E, Lee AH, Iwakoshi NN, Ozdelen E, Tuncman G, Gorgun C, Glimcher LH, Hotamisligil GS. Endoplasmic reticulum stress links obesity, insulin action, and type 2 diabetes. Science 2004;306:457-61

Schaffer SW, Azuma J, Mozaffari M. Role of antioxidant activity of taurine in diabetes. Can J Physiol Pharmacol 2009; 87:91-9

Segal KR, Landt M, Klein S. Relationship between insulin sensitivity and plasma leptin concentration in lean and obese men. Diabetes 1996;45:988-91

Shulman GI. Cellular mechanisms of insulin resistance. J Clin Invest 2000;106:171-6

Tokunaga H, Yoneda Y, Kuriyama K. Streptozotocin-induced elevation of pancreatic taurine content and suppressive effect of taurine on insulin secretion. Eur J Pharmacol 1983; 87:237-43

Wang LJ, Yu YH, Zhang LG, Wang Y, Niu N, Li Q, Guo LM. Taurine rescues vascular endothelial dysfunction in streptozocin-induced diabetic rats: correlated with downregulation of LOX-1 and ICAM-1 expression on aortas. Eur J Pharmacol 2008;597:75-80

Warnick GR, Albers JJ. A comprehensive evaluation of the heparin-manganese precipitation procedure for estimating high density lipoprotein cholesterol. J Lipid Res 1978;19: 65-76

Yamauchi T, Kamon J, Waki H, Terauchi Y, Kubota N, Hara K, Mori Y, Ide T, Murakami K, Tsuboyama-Kasaoka N, Ezaki O, Akanuma Y, Gavrilova O, Vinson C, Reitman ML, Kagechika H, Shudo K, Yoda M, Nakano Y, Tobe K, Nagai R, Kimura S, Tomita M, Froguel P, Kadowaki T. The fat-derived hormone adiponectin reverses insulin resistance associated with both lipoatrophy and obesity. Nat Med 2001;7:941-6

Yamauchi T, Kamon J, Minokoshi Y, Ito Y, Waki H, Uchida S, Yamashita S, Noda M, Kita S, Ueki K, Eto K, Akanuma Y, Froguel P, Foufelle F, Ferre P, Carling D, Kimura S, Nagai R, Kahn BB, Kadowaki T. Adiponectin stimulates glucose utilization and fatty-acid oxidation by activating AMPactivated protein kinase. Nat Med 2002;8:1288-95 